DOE DE-FG26-99FT-40584

PROGRESS REPORT

October 2001

\title{
ADVANCED COMPUTATIONAL MODEL FOR THREE-PHASE SLURRY REACTORS
}

Grant Number: DE-FG26-99FT-40584

Goodarz Ahmadi

Department of Mechanical and Aeronautical Engineering Clarkson University

Submitted to

U.S. Department of Energy

National Energy Technology Laboratory 
TITLE:

\section{ADVANCED COMPUTATIONAL MODEL FOR THREE-PHASE SLURRY REACTORS}

PI:

Goodarz Ahmadi

STUDENTS: $\quad$ X. Zhang, A. R. Mazaheri, H. Zhang, C. He, J. Cao, W. Kvasnak

INSTITUTION: Clarkson University

Potsdam, NY 13699-5725

Tel: (315) 268-2322

Fax: (315) 268-6438

Email: ahmadi@clarkson.edu

GRANT NO.: $\quad$ DE-FG2699-FT40458

\section{PERIOD OF}

PERFORMANCE: September 1, 1999 to August 31, 2002

DOE Project Officers: Dr. Donald Krastman

\section{SUMMARY}

In the second year of the project, the Eulerian-Largrangian formulation for analyzing three-phase slurry flows in a bubble column is further developed. The approach uses an Eulerian analysis of liquid flows in the bubble column, and makes use of the Lagrangian trajectory analysis for the bubbles and particle motions.

An experimental set for studying a two-dimensional bubble column is also developed. The operation of the bubble column is being tested and diagnostic methodology for quantitative measurements is being developed.

An Eulerian computational model for the flow condition in the two-dimensional bubble column is also being developed. The liquid and bubble motions are being analyzed and the results are being compared with the experimental setup.

Solid-fluid mixture flows in ducts and passages at different angle of orientations were analyzed. The model predictions were compared with the experimental data and good agreement was found. Gravity chute flows of solid-liquid mixtures is also being studied. 
Further progress was also made in developing a thermodynamically consistent model for multiphase slurry flows with and without chemical reaction in a state of turbulent motion. The balance laws are obtained and the constitutive laws are being developed.

Progress was also made in measuring concentration and velocity of particles of different sizes near a wall in a duct flow. The technique of Phase-Doppler anemometry was used in these studies.

\section{OBJECTIVES}

The general objective of this project is to provide the needed fundamental understanding of three-phase slurry reactors in Fischer-Tropsch (F-T) liquid fuel synthesis. The other main goal is to develop a computational capability for predicting the transport and processing of three-phase coal slurries. The specific objectives are:

\$ To develop a thermodynamically consistent rate-dependent anisotropic model for multiphase slurry flows with and without chemical reaction for application to coal liquefaction. Also establish the material parameters of the model.

\$ To provide experimental data for phasic fluctuation and mean velocities, as well as the solid volume fraction in the shear flow devices.

\$ To develop an accurate computational capability incorporating the new rate-dependent and anisotropic model for analyzing reacting and nonreacting slurry flows, and to solve a number of technologically important problems related to Fischer-Tropsch (F-T) liquid fuel production processes.

$\$ \quad$ To verify the validity of the developed model by comparing the predicted results with the performed and the available experimental data under idealized conditions.

\section{SIGNIFICANCE TO FOSSIL ENERGY PROGRAM}

Converting coal to liquid hydrocarbon fuel by direct and indirect liquefaction processes has been of great concern to the development of coal-based energy systems. While the direct hydrogenation has been quite successful and was further developed in various forms, use of slurry phase Fischer-Tropsch (F-T) processing is considered a potentially more economical scheme to convert synthesis gas into liquid fuels. Slurry transport and processing and pneumatic transport of particles play a critical role in the operation, efficiency, safety and maintenance of these advanced coal liquefaction and coal-based liquid fuel production systems. 
Therefore, a fundamental understanding of reacting coal slurries will have a significant impact on the future of environmentally acceptable liquid fuel generation from coal.

Particle-particle and particle-gas/liquid interactions strongly affect the performance of three-phase slurry reactors used in coal conversion processes and are crucial to the further development of coal-based synthetic hydrocarbon fuel production systems. The scientific knowledge base for these processes, however, is in its infancy. Therefore, most current techniques were developed on an $a d$ hoc and trial and error basis. This project is concerned with for providing the needed fundamental understanding of the dynamics of chemically active slurries and three-phase mixtures. In particular, a computational model for predicting the behavior of dense mixtures in coal liquefaction and liquid fuel production equipment will be developed.

\section{PROGRESS REPORT}

\section{GENERAL}

Progress was made in various aspect of the research project. The highlight of the accomplishment is first summarized. This is followed by a description of the progress made. One important additional accomplishment of the project is the development of an experimental setup for a two-dimensional bubble column. This additional effort, which was not in the scope of the original proposed work, was undertaken to provide quantitative data for our model verification.

\section{HIGHLIGHT OF ACCOMPLISHMENTS}

An Eulerian-Largrangian formulation for analyzing three-phase slurry flows in a bubble column is being developed. The approach uses an Eulerian analysis of liquid flows in the bubble column, and makes use of the Lagrangian trajectory analysis for the motions of bubbles and particles. The developed method accounts for the two-way interactions of the three-phase flows.

A computational model for two-phase flow was developed and the flows in horizontal and inclined ducts were analyzed. The results were compared with the available experimental data and earlier model predictions and good agreements were observed. A computational model for analyzing two-phase solid-liquid flows at various mass loading ratios was also developed and was successfully used to predict flow parameters down an inclined chute. 
An experimental set-up for generating a two-dimensional bubble column for detail studies is fabricated. The diagnostic methods for quantitative measurements are being developed.

Progress was also made in studying two-phase bubbly flows using the volume-of-fluid (VOF) approach. The Largragian trajectory of a dilute concentration of the solid phase is also studied. In a related modeling effort, progress was made in developing a rate dependent thermodynamically consistent model for slurry flows. The new model includes the effect of phasic interactions and appears to lead to anisotropic effective stress tensor. The formulation is being extended to cover three-phase liquid-gas-solid flows.

Progress was also made in analyzing turbulent two-phase flows with heat transfer. We developed an Eulerian/Lagrangian approach including the two-way interaction for two-phase flows. The model considers the thermal turbulent field characteristics and includes an explicit equation for temperature fluctuation in addition to the turbulence kinetic energy and time scales of the flow and thermal field fluctuations.

Progress was also made in measuring concentration and velocity profiles of particles of different sizes near a solid wall in a duct flow. The result shows that small particles have diffusion dominated concentration profiles near the wall, while the larger particles could acquire an inertial dominated counter gradient profile.

\section{EULERIAN-LAGRANGIAN MODEL FOR THREE-PHASE SLURRY FLOWS}

A computational model for three phase slurry flows is developed. The continuous liquid phase is be modeled using an Eulerian formulation. The discrete bubbles and solid particles are treated with a Lagrangian trajectory tracking approach. The model accounts for the two-way interactions between the phases. Figure 1 shows a sample computed flow field in a box 0.03 $s$ after the initiation of the flow. Here the flow starts form an initial condition that particles and bubbles are uniformly distributed in the liquid. In this figure particles are identified by darker (red) dots, while the bubbles are lighter (green) circles. Figure 2 shows the computed flow field at $\mathrm{t}=0.09 \mathrm{~s}$. It is seen that the bubbles are moving rapidly upward due to their large buoyancy forces. The small solid particles are transported by the flow stream, while are drifted downward due to the gravitational sedimentation. 


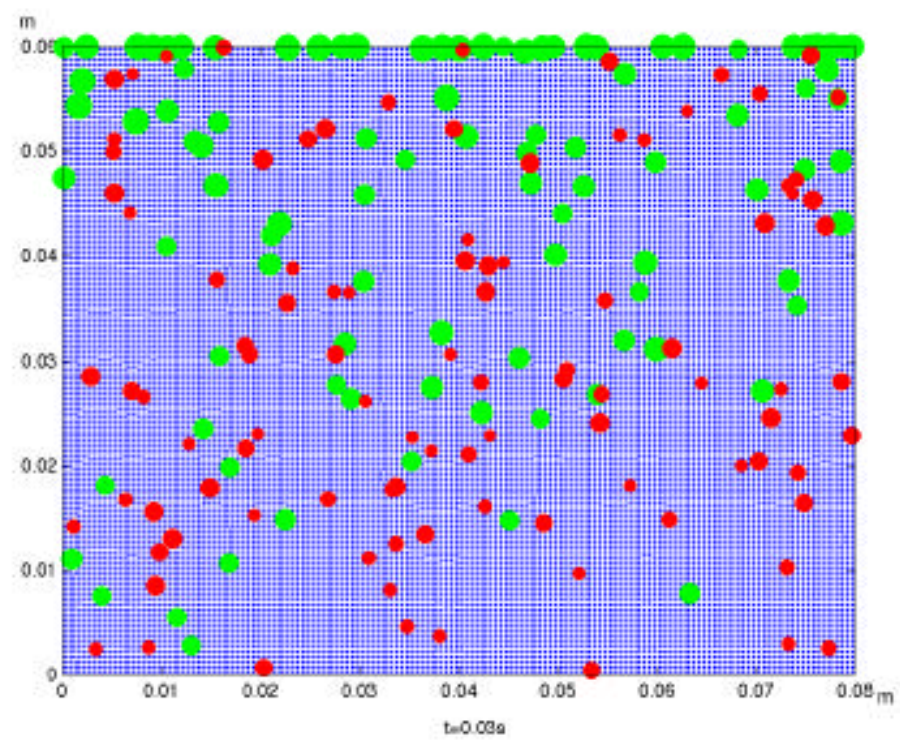

Figure 1. Configuration of the flow in a box at $t=0.03 \mathrm{~s}$.

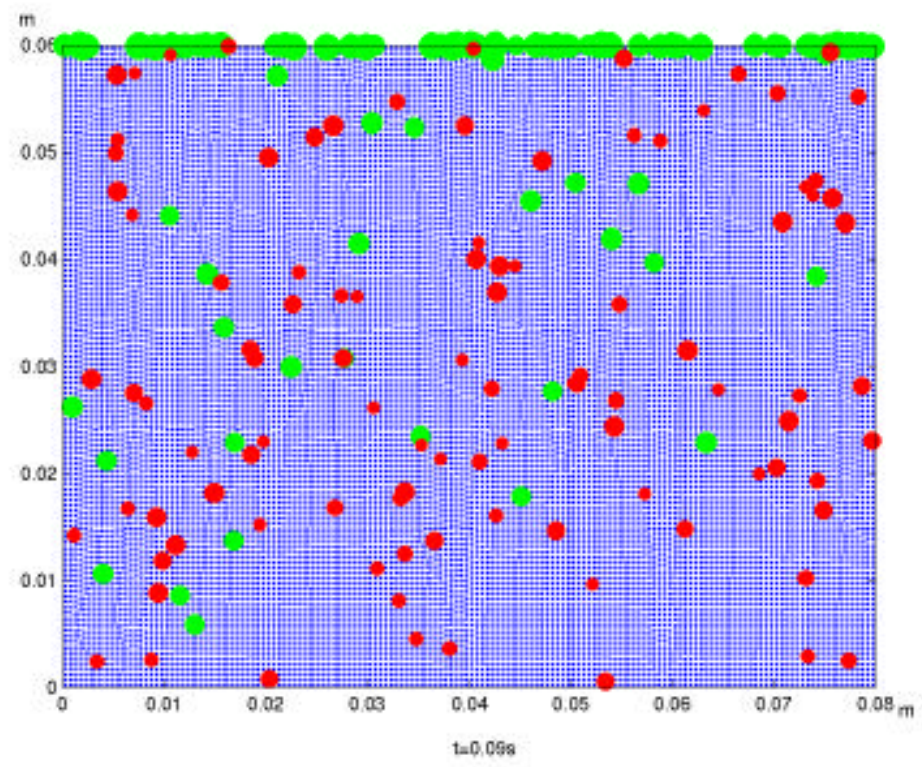

Figure 2. Configuration of the flow in a box at $t=0.09 \mathrm{~s}$. TWO-PHASE FLOWS IN A DUCT WITH HEAT TRANSFER 
The problem of two-phase flows with heat transfer in a duct is studied. The formulation of two-phase flows in a duct is presented in this section. The model accounts for the two-way interactions both in moment and energy transport.

\section{Hydrodynamic Formulation}

The equations of motion of turbulent flow field are obtained by applying the Reynolds decomposition on the instantaneous momentum equation. The closed time-dependent equation for the mean gas velocity in a vertical fully developed axisymmetric gas-solid flow in cylindrical coordinates is given as

$$
\frac{D\left[(1-\phi) U_{g}\right]}{D t}=\frac{1}{r} \frac{\partial}{\partial r}\left[r\left(v+v_{t}\right)(1-\phi) \frac{\partial U_{g}}{\partial r}\right]-(1-\phi) g_{o}-\frac{(1-\phi)}{\rho_{g}} \frac{\partial p}{\partial x}+S_{u}
$$

Here $\phi$ is the solid volume fraction, $S_{u}$ is the coupling source term due the interaction of gas and solid, and $v_{t}=c_{\mu} f_{\mu} k \tau$, is the eddy viscosity, where $c_{\mu}=0.09$, and $f_{\mu}$ is the damping function. In Equation (1), $S_{u}$, the coupling source term due to the presence of particle which is given as

$$
S_{u}=\frac{\rho_{p}}{\tau_{p} \rho_{g}}\left[\phi_{p}\left(U_{p}-U_{g}\right)\right]
$$

where $\tau_{p}$ is the particle dynamic relaxation time, defined as

$\tau_{p}=\frac{4 \quad \rho_{p} d_{p}}{3 \rho_{g} C_{D}\left|U_{p}-U_{g}\right|}$

and $C_{D}$ is the Drag coefficient.

For an axisymmetric fully developed two-phase flow, the resulting $k-\tau$ transport equations are given as

$$
\begin{aligned}
& \frac{D(1-\phi) k}{D t}=\frac{1}{r} \frac{\partial}{\partial r}\left[r(1-\phi)\left(N+\frac{v_{t}}{\sigma_{k}}\right) \frac{\partial k}{\partial r}\right]+v_{t}(1-\phi)\left(\frac{\partial U_{g}}{\partial r}\right)^{2}-(1-\phi) \varepsilon-S_{k} \\
& \frac{D(1-\phi) \tau}{D t}=\frac{1 \partial}{r \partial r}\left[r(1-\phi)\left(v+\frac{v_{t}}{\sigma_{\tau}}\right) \frac{\partial \tau}{\partial r}\right]+c_{\tau 1}(1-\phi)-c_{\tau 2}(1-\phi) \frac{\tau}{k} v_{t}\left(\frac{\partial U_{g}}{\partial r}\right)^{2}
\end{aligned}
$$




$$
+\frac{2}{k}(1-\phi)\left[\left(v+\frac{\tau_{t}}{\sigma_{\tau}}\right) \frac{\partial \tau \partial k}{\partial r \partial r}\right]-\frac{2}{\tau}(1-\phi)\left[\left(v+\frac{v_{t}}{\sigma_{\tau}}\right) \frac{\partial \tau \partial \tau}{\partial r \partial r}\right]+S_{\tau}
$$

The coefficients in Equations (4) and (5) are given as $c_{\tau 1}=0.92, c_{\tau 2}=0.44, \sigma_{k}=1$, and $\sigma_{\tau}=1$. In Equation (4), $S_{k}$ is the source term due to the solid phase interaction with gas. i.e.,

$$
\left.S_{k}=-\frac{\rho_{p}}{2 \rho_{g} \tau_{p}}\left[\bar{\phi}\left(\overline{u_{i}^{p^{\prime}} u_{i}^{g^{\prime}}}-\overline{u_{i}^{g^{\prime}} u_{i}^{g^{\prime}}}\right)+\overline{\phi^{\prime} u_{1}^{g^{\prime}}}\left(U_{p}-U_{g}\right)\right]-\frac{\rho_{p}}{2 \rho_{g} \tau_{p}} \overline{\phi^{\prime} u_{i}^{g^{\prime}}\left(u_{i}^{p^{\prime}}-u_{i}^{g^{\prime}}\right)}\right]
$$

Here $u_{i}^{g^{\prime}}$ and $u_{i}^{p^{\prime}}$ are, respectively, the fluctuation velocities of gas and particle phases, and $\phi^{\prime}$ is the particle concentration fluctuation. Neglecting the triple correlation terms, the coupling term becomes

$\left.S_{k}=-\frac{\rho_{p}}{2 \rho_{g} \tau_{p}}\left[\bar{\phi} \overline{\left(u_{i}^{p^{\prime}} u_{i}^{g^{\prime}}\right.}-\overline{u_{i}^{g^{\prime}} u_{i}^{\prime g}}\right)+\overline{\phi^{\prime} u_{1}^{\prime g}}\left(U_{p}-U_{g}\right)\right]$

The source term in the Equation (5) is given as

$S_{\tau}=\frac{S_{k}}{\varepsilon}\left(c_{\tau 3}-1\right)$

Here, coefficient $c_{\tau 3}=2.0$ is used.

The gas-particle velocity correlation term $\overline{u_{i}^{p^{\prime}} u_{i}^{g^{\prime}}}$ in Equation (7) is evaluated using a combined Eulerian-Lagrangian averaging procedure. First the mean particle velocity, $U_{p}=U_{1}^{p}$, during each Lagrangian time step is evaluated within a computational control volume (computational cell) around each node by ensemble averaging. Then the cross correlation term $\overline{u_{i}^{p^{\prime}} u_{i}^{g^{\prime}}}$ is evaluated by the following averaging procedure:

$$
\overline{u_{i}^{p^{\prime}} u_{i}^{g^{\prime}}}=\frac{1}{\Delta t_{E} N_{p}} \sum_{k=1}^{N_{t}} \sum_{n=1}^{N_{p}}\left[\left(u_{i}^{p}-U_{i}^{p}\right)\left(u_{i}^{g}-U_{i}^{g}\right)\right] \Delta t_{L}
$$

where $u_{i}^{p}$ is the instantaneous particle velocity, and $u_{i}^{g}$ is the instantaneous gas velocity. Here, $N_{p}$ is the number of particle in the computational cell, $\Delta t_{E}=N_{t} \cdot \Delta t_{L}$ and $N_{t}$ is the number of Lagrangian time steps in each Eulerian time step. In Equation (9), the summation over n (and division by $N_{P}$ ) indicates the ensemble averaging over the particles in each computational cell, and the summation over $\mathrm{k}$ (and division by $N_{t}$ ) denotes the temporal averaging over theEulerian time step.

The particle concentration- gas velocity correlation term is modeled using a gradient 
transport hypothesis. That is, $\overline{\phi^{\prime} u_{1}^{\prime g}} \approx \frac{v_{t} \partial \bar{\phi}}{\sigma_{p g} \partial r}$, where, $\sigma_{p g}$ is taken to be a constant equal to 1.

\section{Thermal Formulation}

The equation governing the mean turbulent gas temperature is given as

$$
\frac{D(1-\phi) T}{D t}=\frac{1}{r} \frac{\partial}{\partial r}\left[r(1-\phi)\left(\alpha+\alpha_{t}\right) \frac{\partial T}{\partial r}\right]+S_{T}
$$

The thermal eddy diffiusivity is by $\alpha_{t}=c_{\lambda} f_{\lambda} k \tau_{\theta}$. Here $\tau_{\theta}$ represents thermal time scale, $\mathrm{c}_{\lambda}$ is a constant, which is assumed to be equal to 0.2 , and $f_{\lambda}$ is a damping function. The second term on the right hand side of Equation (10) is the coupling term due to the solid phase interaction with the gas. i.e.,

$$
S_{T}=\frac{F}{\rho_{g} c_{p g}}\left[\phi\left(T_{p-} T_{g}\right)\right]
$$

where $F=6 N u_{p} K_{g} / d_{p}^{2}$.

The resulting $k_{\theta}-\tau_{\theta}$ transport equations for an axisymmetric fully developed two-phase flow are given as

$$
\begin{aligned}
\frac{D(1-\phi) k_{\theta}}{D t}=\frac{1}{r} & \frac{\partial}{\partial r}\left[r(1-\phi)\left(\alpha+\frac{\alpha_{t}}{\sigma_{k \theta}}\right) \frac{\partial k_{\theta}}{\partial r}\right]+\alpha_{t}\left(\frac{\partial T_{g}}{\partial r}\right)^{2}-\varepsilon_{\theta}-S_{k \theta} \\
\frac{D(1-\phi) \tau_{\theta}}{D t}= & \frac{1 \partial}{r \partial r}\left[r(1-\phi)\left(\alpha+\frac{\alpha_{t}}{\sigma_{\tau \theta}}\right) \frac{\partial \tau_{\theta}}{\partial r}\right]+c_{\tau \theta 1}(1-\phi) \frac{\tau_{\theta}}{k_{\theta}} \alpha_{t}\left(\frac{\partial T_{g}}{\partial r}\right)^{2}+c_{\tau \theta 2}(1-\phi) \frac{\tau_{\theta}}{k} v_{t}\left(\frac{\partial U_{g}}{\partial r}\right)^{2} \\
& +\left(c_{\tau \theta 3}-1\right)(1-\phi)+\frac{2}{k_{\theta}}(1-\phi)\left[\left(\alpha+\frac{\alpha_{t}}{\sigma_{\tau \theta}}\right) \frac{\partial \tau_{\theta} \partial k_{\theta}}{\partial r}\right]-\frac{2}{\tau_{\theta}}(1-\phi)\left[\left(\alpha+\frac{\alpha_{t}}{\sigma_{\tau \theta}}\right) \frac{\partial \tau_{\theta} \partial \tau_{\theta}}{\partial r}\right] \partial r \\
& +c_{\tau \theta 4}(1-\phi) \frac{\tau_{\theta}}{\tau}+S_{\tau \theta}
\end{aligned}
$$

The model coefficients in Equations (12) and (13) are

$c_{\tau \theta 1}=0.27, c_{\tau \theta 2}=-0.7, c_{\tau \theta 3}=\left[1-\exp \left(-y^{+} / 4.8\right)\right]^{2}, c_{\tau \theta 4}=(1.92-1)\left[1-\exp \left(-y^{+} / 4.9\right)\right]^{2}$ 
In Equation (12), $S_{k \theta}$, is the source term due to the solid phase interaction with gas, and is given by

$$
S_{k \theta}=-\frac{F}{2 \rho_{g} c_{p g}}\left[\bar{\phi}\left(\overline{\overline{t_{p} t_{g}^{\prime}}}-\overline{t_{g}^{\prime} t_{g}^{\prime}}\right)+\overline{\phi^{\prime} \overline{t g}_{g}^{\prime}}\left(T_{p}-T_{g}\right)\right]
$$

where $t_{g}^{\prime}$ is the fluctuating gas temperature and $t_{p}^{\prime}$ is the fluctuating particle temperature. The correlation terms between particle concentration and gas temperature are modeled by a gradient transport hypothesis given as $\overline{\phi^{\prime} t_{g}^{\prime}} \approx \frac{\alpha_{t} \partial \bar{\phi}}{\sigma_{t g} \partial r}$, where, $\sigma_{t g}=1$ is a constant.

The gas-particle temperature correlation term $\overline{\overline{t_{p} t_{s}^{\prime}}}$ in Equation (14) is evaluated using a combined Eulerian-Lagrangian averaging procedure similar to that used for the gas-particle velocity correlation. First the mean particle temperature, $T_{p}$, during each Lagrangian time step is evaluated within a computational control volume (computational cell) around each node by ensemble averaging. Then the cross correlation $\overline{t_{p} t_{g}^{\prime}}$ is evaluated by ensemble averaging over the particles in each computational cell and temporal averaging over the Eulerian time step similar to the procedure described for the velocity cross correlation.

\section{Instantaneous Turbulence Fluctuation}

The generation of fluctuating components of fluid velocity using a continuous Gaussian random field model was suggested by Kraichnan. Accordingly, the fluctuation component of the turbulence in an isotropic field is given by

$\vec{u}^{\prime+}\left(\vec{X}^{+}, t^{+}\right)=\sqrt{\frac{2}{M}}\left\{\sum_{n}^{M} \vec{U}_{1}\left[\cos \left(\vec{K}_{n} \cdot \vec{X}^{+}+\omega_{n} t^{+}\right)\right]\right\}+\sqrt{\frac{2}{M}}\left\{\sum_{n}^{M} \vec{U}_{2}\left[\sin \left(\vec{K}_{n} \cdot \vec{X}^{+}+\omega_{n} t^{+}\right)\right]\right\}$

where $\vec{X}^{+}$is the position vector and all quantities are nondimensionalized with a velocity scale $u^{*}$ and kinematic viscosity. That is

$u_{i}^{+}=\frac{u_{i}}{u^{*}}, t^{+}=\frac{t u^{* 2}}{v}, x_{i}^{+}=\frac{x_{i} u^{*}}{v}$

In Equation (15)

$\vec{U}_{1}=\vec{\zeta}_{n} \times \vec{K}_{n}, \quad \vec{U}_{2}=\vec{\xi}_{n} \times \vec{K}_{n}, \quad \vec{K}_{n} \cdot \vec{U}_{1}=\vec{K}_{n} \cdot \vec{U}_{2}=0$. 
The components of vectors $\vec{\varsigma}_{n}, \vec{\xi}_{n}$, and frequencies $\omega_{n}$ are picked independently from a Gaussian distribution with a standard deviation of unity. Each component of $\vec{K}_{n}$ is also a Gaussian random number with a standard deviation of $1 / 2$. In Equation (20) $\mathrm{M}$ is the number of terms in the series. (Here $\mathrm{M}=100$ is used.)

Equation (15) generates a continuous incompressible Gaussian random field, which resembles an isotropic homogeneous turbulence. For application to nonhomogeneous flows a scaling is needed. Here a similar scaling is used using the available data for turbulent velocity field.

The approach is also extended and used for generating the temperature fluctuations. That is, the nondimensional fluctuation temperature is evaluated from

$$
t^{++}\left(\vec{X}^{+}, t^{+}\right)=\sqrt{\frac{2}{M}}\left\{\sum_{n}^{M} T_{1}\left[\cos \left(\vec{K}_{n} \cdot \vec{X}^{+}+\omega_{n} t^{+}\right)\right]\right\}+\sqrt{\frac{2}{M}}\left\{\sum_{n}^{M} T_{2}\left[\sin \left(\vec{K}_{n} \cdot \vec{X}^{+}+\omega_{n} t^{+}\right)\right]\right\}
$$

Here $\mathrm{t}^{\prime+}=\mathrm{t}_{\mathrm{g}}^{\prime} / \mathrm{t}^{*}$ with $\mathrm{t}^{*}$ being the root-mean square gas temperature fluctuation. All random coefficients in Equation (18) are generated similar to that of the Kraichnan model for the flow field, except for $T_{1}$ and $T_{2}$ that are picked from independent Gaussian distributions with a standard deviation of unity.

\section{Particle Lagrangian Simulation}

A Lagrangian particle tracking approach is used in the analysis. The equation of motion for a spherical particle including the viscous drag and gravitational forces is given by

$$
\begin{aligned}
\frac{d u_{i}^{p}}{d t} & =\frac{3 C_{d} \rho_{g}}{4 d_{p} \rho_{p}}\left|u_{i}^{g}-u_{i}^{p}\right|\left(u_{i}^{g}-u_{i}^{p}\right)+g \\
\frac{d x_{i}^{p}}{d t} & =u_{i}^{p}
\end{aligned}
$$

Here $u_{i}^{p}$ is the particle velocity, $u_{i}^{g}$ is the instantaneous fluid velocity with $u_{i}^{g}=U_{i}^{g}+u_{i}^{g^{\prime}}$, where $U_{i}^{g}$ is the fluid mean velocity and $u_{i}^{g}$ is the fluctuating component. In Equation (19) $\mathrm{C}_{\mathrm{d}}$ is the local drag coefficient, which is a function of particle Reynolds number.

When a particle strikes a wall, it is assumed that it will bounce from the surface. The rebound velocity of a solid particle from the wall is evaluated using the classical impulse equation for inelastic collisions. Here unless stated otherwise a coefficient of restitution of 
0.7 in used.

Thermal energy equation of the particles is given as

$m c{ }_{p} \frac{d T^{p}}{d t}=h_{p} A\left(T^{g}-T^{p}\right)$

where $\mathrm{m}$ is the particle mass, $\mathrm{c}_{\mathrm{p}}$ is the particle heat capacity, $\mathrm{A}$ is the particle surface area and $\mathrm{h}_{\mathrm{p}}$ is the heat transfer coefficient. The term on the right hand side of (21) is due to the gasparticle heat transfer. Here $T^{p}$ is the particle temperature, and $T^{g}$ is the fluid temperature at the particle location. Note that $T^{g}=T_{i}^{g}+t^{g}$, where $t^{g^{\prime}}$ is the gas fluctuating temperature, which is generated using the extended Kraichnan model given by Equation (18). The convection transfer coefficient $h_{p}$ is given as

$N u_{p}=\frac{h_{p} d}{k_{g}}=2+0.6 \operatorname{Re}_{p}^{0.5} \operatorname{Pr}^{0.3}$

$N u_{p}$ is the Nusselt number, $k_{g}$ is the gas heat conductivity, and $\operatorname{Pr}$ is the Prandtl number.

It should be emphasized that the mean gas velocity and temperature are evaluated from the Eulerian field equations. The instantaneous gas velocity and temperature at the particle location are then determined by adding the fluctuation fields as given by Equations (15) and (18) to the mean fields. The instantaneous values are then used in Equations (19) and (21) for evaluating the particle motion and temperature.

\section{TWO-PHASE FLOWS IN HORIZONTAL AND INCLINED DUCTS}

Using the earlier developed thermodynamically consistent model, a computational procedure for solving dense and dilute two-phase flows in ducts at various angle was developed. The computational model predictions for mean flow and particle velocities, and phasic turbulence intensities were evaluated and the results are compared with the experimental data of Tsuji et al. (1989) for a horizontal duct flow. In addition, the variations of phasic shear and normal stresses, as well as the phasic fluctuation energy production and dissipation were also evaluated. The part of the study is completed. 


\section{EXPERIMENTAL STUDY OF BUBBLE COLUMN}

An experimental setup for a two-dimensional babble column is designed and fabricated. The setup is $80 \mathrm{~cm}$ high and $40 \mathrm{~cm}$ wide with a thickness of $1 \mathrm{~cm}$, and is made of Plexiglas. The gas distributor is made up of five tube injectors flush mounted on the bottom plate of the column. Each gas injector opening is 1/8 inch, generating an initial bubble size of about 5 8 $\mathrm{mm}$. The distance between two adjacent injectors is $7 \mathrm{~cm}$ and that between the end injector and the sidewall is $6 \mathrm{~cm}$. Air is used as the gas phase. The gas flows through each injector individually from a mixing chamber, which is regulated by a flowmeter. The superficial gas velocity ranges from 5 to $60 \mathrm{~cm} / \mathrm{s}$. Tap water is used as the liquid phase. The liquid phase is operated under batch condition and the static liquid height is kept constant at $70 \mathrm{~cm}$ for all runs. A schematics of the setup is shown in Figure 3.

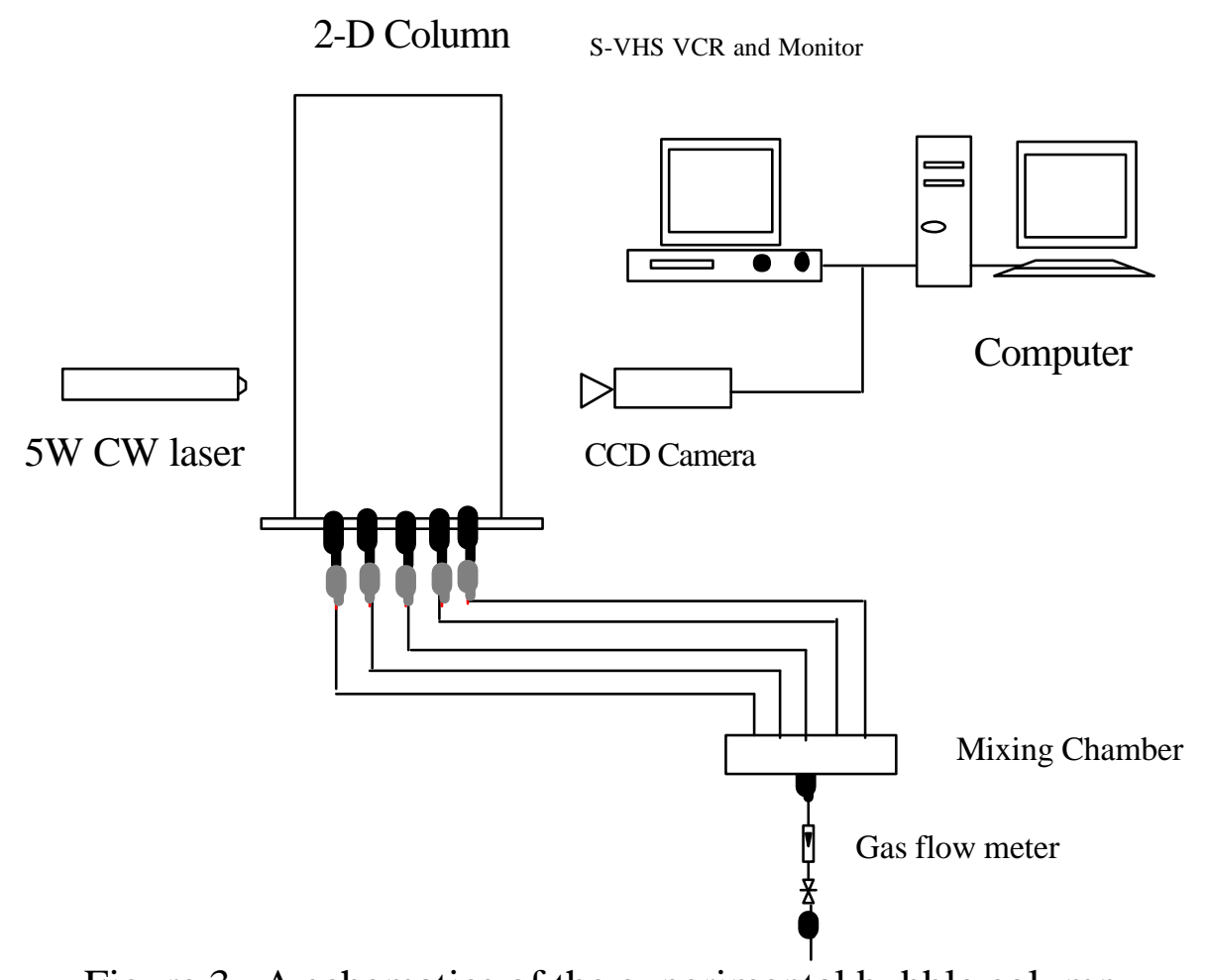

Figure 3. A schematics of the experimental bubble column.

Figure 4 shows a picture of the vessel. We plan to make use of our PIV system to make measurements of the liquid flow and bubble motion in the vessel. 


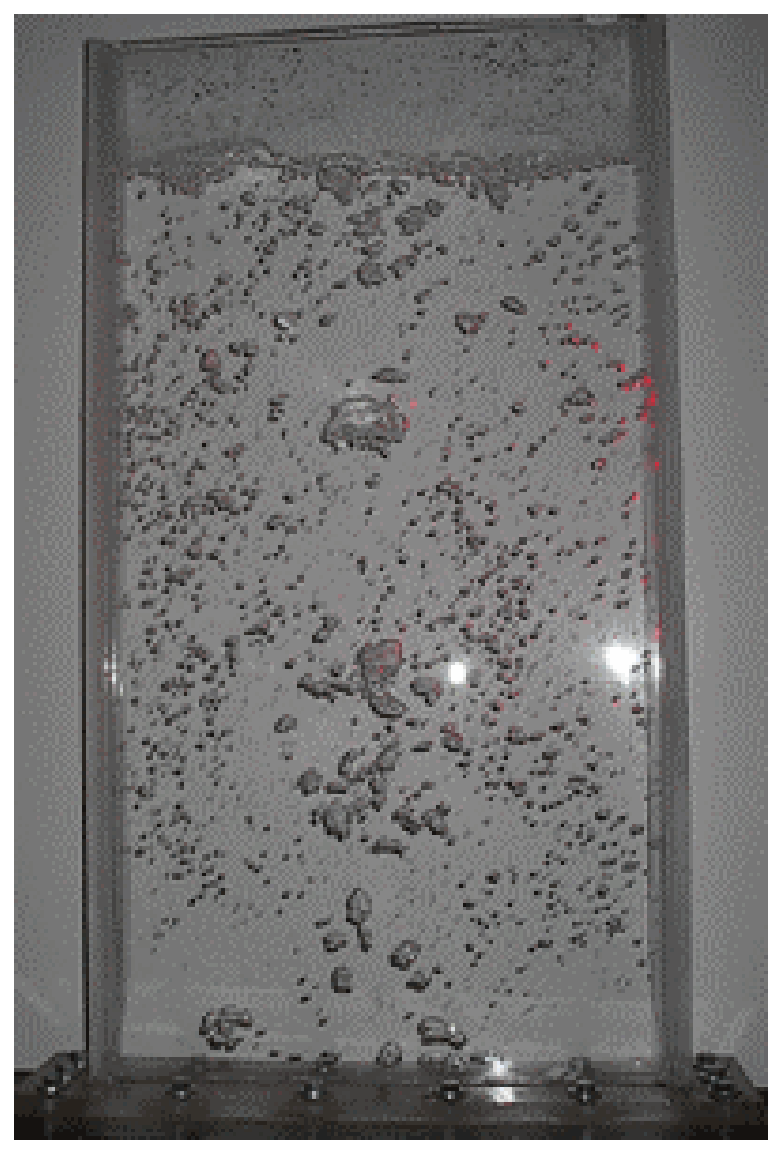

Figure 4. A picture of the experimental bubble column.

\section{VOF ANALYSIS OF BUBBLE COLUMN}

A VOF (volume-of-fluid) analysis of the experimental bubble column is being developed. The FLUENT code is used to evaluate the unsteady liquid-bubble flows in the bubble column. The preliminary results of this approach are described in this section. The height of the column is $0.8 \mathrm{~m}$ with a width of $40 \mathrm{~cm}$. Air enters from five 2-mm holes at the bottom of the column. Figure 5 shows the concentration contours in the column at $1.1 \mathrm{~s}$ after the startup. (The gravity points from right to left in this figure.) The initial stages of air bubble formation are observed from this figure. 


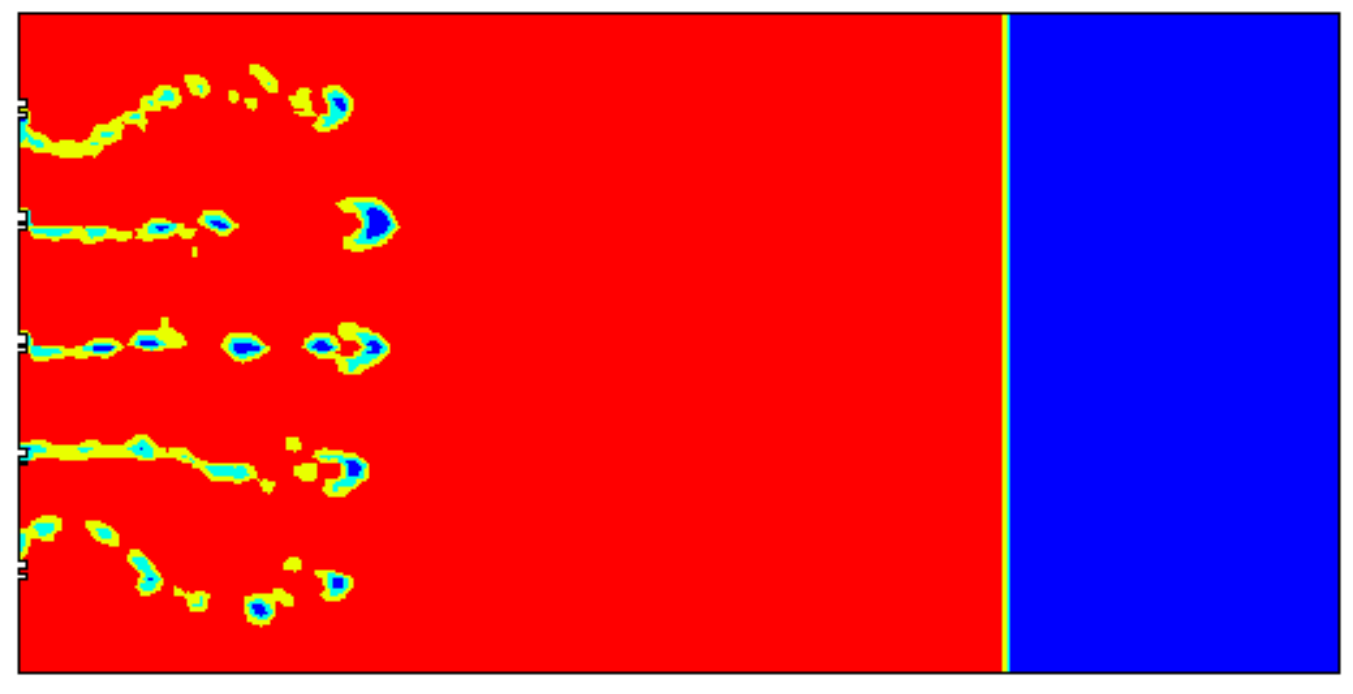

Figure 5. Concentration contours in the bubble column at $\mathrm{t}=1.1 \mathrm{~s}$.

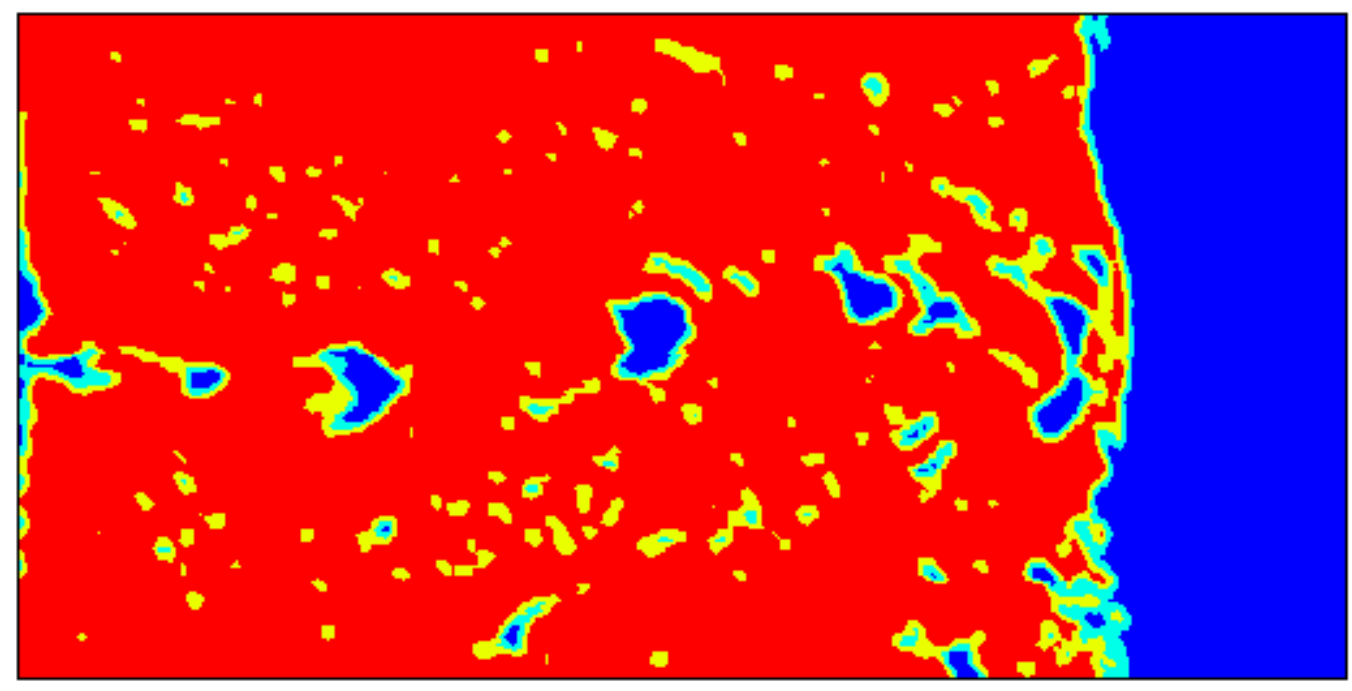

Figure 6. Concentration contours in the bubble column at $\mathrm{t}=6 \mathrm{~s}$. 
The concentration profile after 6 seconds when the flow is shown in Figure 6 . It is seen that the flow in the bubble column is well developed. The bubbly motions also generate rather complex flow pattern in the column. While the detail measurements for the experimental setup is not as yet available, the general features of the computed bubble concentration in Figure 6 is comparable with that observed in Figure 4.

\section{EXPERIMENTAL STUDY NEAR THE WALL}

The earlier research provided a significant level of understanding of the process of particulate transport and deposition processes in turbulent flows. Accordingly, particles are transported by the mean flow field and are dispersed by turbulent diffusion. Near the wall, particles are entrained in the coherent wall vortices, which are the dominant flow structure in the sublayer. The downflow region of the vortices transport the particles to the wall. We performed an experimental study of the particle concentration profile and velocity characteristics in the near wall region of a turbulent channel flow. Measurements are made up to very short distances from the channel wall. The nonintrusive, technique of Phase-Doppler Anemometry is used in the experimentation. The technique allows for the simultaneous measurement of particle size and velocity.

The experimental setup is a horizontal wind tunnel consists of a rectangular duct with a cross sectional area of $15.25 \times 2.54 \mathrm{~cm}^{2}{ }^{2}$ The width to height aspect ratio of about six provides a roughly two-dimensional flow condition near the centerline of the channel. The channel is $3.2 \mathrm{~m}$ long, and the test section, which is $0.36 \mathrm{~m}$ long, is $2.36 \mathrm{~m}$ from the tunnel contraction section. The windows of the test section are made of fused silica optical glass. The tunnel provides the 50 hydraulic diameters needed to ensure a fully developed air velocity profile at the test section. The channel is made of aluminum and is electrically grounded, which provides a hydraulically smooth static charge free surface. A picture of the experimental setup is shown in Figure 9.

\section{CHEMICALLY ACTIVE TWO-PHASE FLOWS}

Progress is made in the formulation of a thermodynamically consistent model for chemically active multiphase flows. The equations governing the phasic conservation of mass and energy, as well as the balance of momentum and fluctuation energy are derived. The appropriate form of the mean entropy inequality is formulated. The entropy equation is being used for formulating thermodynamically consistent constitutive equations for chemically active multi-phase mixtures in a turbulent state of motion in the bubble column. 


\section{PLANS FOR THE COMING YEAR}

$\$ \quad$ To perform detailed experimentation of the bubble column in two and three phase operation mode.

$\$$ To develop a detailed computational model for predicting the operation of the bubble column and three phase flows.

\$ To complete the thermodynamically consistent model for three-phase multiphase slurry flows in bubble columns.

$\$ \quad$ To evaluate the model parameters for the cases of practical interest to liquid fuel production from coal.

\$ To simulate technologically important problems related to Fischer-Tropsch (F-T) liquid fuel production processes.

$\$$ To perform experimental measurements of the phasic properties in the simple shear flow device.

\section{ARTICLES, PRESENTATIONS AND STUDENT SUPPORT}

\section{Journals Articles (peer reviewed)}

He, C. and Ahmadi, G., Particle Deposition in a Nearly Developed Turbulent Duct Flow with Electrophoresis, J. Aerosol Science, Vol. 30, pp. 739-758 (1999).

Cao, J. and Ahmadi, G., Gas-Particle Two-Phase Flow in Horizontal and Inclined Ducts, Int. J. Engng. Sci, Vol. 38, pp. 1961-1981 (2000).

Zhang, H. and Ahmadi, G., Aerosol Particle Transport and Deposition in Vertical and Horizontal Turbulent Channel Duct Flows, J. Fluid Mechanics, Vol. 406, pp. 55-88 (2000).

Fan, F-G. and Ahmadi, G., Wall Deposition of Small Ellipsoids from Turbulent Air Flows-A Brownian Dynamics Simulation, J. Aerosol Sci., Vol. 31, pp. 1205-1229 (2000).

Soltani, M. and Ahmadi, G., Direct Numerical Simulation of Curly Fibers in Turbulent Channel Flow, Aerosol Science Technology, Vol. 33, pp. 392-418 (2000).

Ahmadi, G., He, C., Ban, H. and Stencel, J.M., Air Flow and Particle Transport in Triboelectric Coal/Ash Cleaning System-Counter Flowing Straight Duct Design, Particulate Science Technology, Vol. 18, pp. 213-256 (2000).

Shams, M., Ahmadi, G. and Rahimzadeh, H., A Sublayer Model for Deposition of Nano- and Micro-Particles in Turbulent Flows, Chemical Engineering Science, Vol. 55, pp. 6097-6107 (2000). 
Zhang, H. and Ahmadi, G., Aerosol Particle Removal and Re-entrainment in Turbulent FlowsA Direct Numerical Simulation Approach, J. Adhesion, Vol. 74, pp. 441-493 (2000).

Zhang, H., Ahmadi, G., Fan, F.-G. and McLaughlin, J.B., Ellipsoidal Particles Transport and Deposition in Turbulent Channel Flows, International Journal Multiphase Flows, Vol. 27, pp. 971-1009 (2001).

Zhang, H. and Ahmadi, G., Particles Transport and Deposition in the Hot-Gas Filter Vessel at Wilsonville, Powder Technology, Vol. 116, pp. 53-68 (2001).

Ahmadi, G. and C. He, Simulation of Particles Transport and Deposition in a Combustor, Chemical Engineering Communication, Vol. 187, pp. $23-53$ (2001).

\section{Conference Presentations}

G. Ahmadi and J. Cao, "Anisotropic Model for Granular and Dense two-Phase Flows," 1999 ASME Mechanics and Materials Conference, Blacksburg, VA, June 27-30, 1999.

G. Ahmadi, K. Elliott and W. Kvasnak, "An Experimental Study of Granular Flow in a Couette Flow Device," 1999 ASME Mechanics and Materials Conference, Blacksburg, VA, June 27-30, 1999.

C. He and G. Ahmadi, "Modeling of Particle Dispersion and Deposition with Thermophoresis in a Controlled Profile Combustor," 18th Annual Conference of the American Association for Aerosol Research, AAAR '99, Tacoma. WA, October 11-15, 1999.

H. Zhang and G. Ahmadi, "Aerosol Particle Removal and Re-entrainment in Turbulent Channel Flows," 18th Annual Conference of the American Association for Aerosol Research, AAAR '99, Tacoma. WA, October 11-15, 1999.

H. Zhang and G. Ahmadi, F. Fan and J.B. McLaughlin, "Analysis of the Motion of Ellipsoidal Particle in Turbulent Channel Flows," 52st Annual Meeting of American Physical Society, Division of Fluid Dynamics, New Orleans, LA, November 21-23, 1999.

P.V. Skudarnov, L.L. Regel, W. R. Wilcox and G. Ahmadi, "Numerical Modeling and Flow Visualization in the Gradient Freeze Configuration During Centrifugation," Fourth International Workshop on Materials Processing at High Gravity, Clarkson University, Potsdam, NY, May 29-June 2, 2000. 
A.R. Mazaheri, H. Zhang and G. Ahmadi, "A Centrifual Filtration Concept for Hot-Gas Cleaning," Fourth International Workshop on Materials Processing at High Gravity, Clarkson University, Potsdam, NY, May 29-June 2, 2000.

G. Ahmadi, "Advanced Computational Model for Three-Phase Slurry Reactors," Abstract and Research Accomplishments of University Coal Research Projects, pp. 91-91, University Coal Research Contractors Review Conference, NETL, Pittsburgh, PA, June 6-7, 2000.

G. Ahmadi and H. Zhang, "Resuspension of Particles in Turbulent Flows," Seventh International Symposium on Particles on Surfaces: Detection, Adhesion and Removal, Newark, NJ, June 1921, 2000.

G. Ahmadi and H. Zhang, "Hot-Gas Flow and Particle Transport and Deposition in the Filter Vessel at Wilsonville," Seventeenth Annual International Pittsburgh Coal Conference, Pittsburgh, PA, September 11-14, 2000.

A.R. Mazaheri and G. Ahmadi, "Computational Modeling of a Centrifual Filtration System," 19th Annual Conference of the American Association for Aerosol Research, AAAR 2000, St. Louis, MO, November 6-10, 2000.

D.J. Schmidt, G. Ahmadi, and G. Schmidt, "Dispersion of Droplets in a Turbulent Spray," 19th Annual Conference of the American Association for Aerosol Research, AAAR 2000, St. Louis, MO, November 6-10, 2000.

M. Shams, G. Ahmadi and H. Rahimzadeh, "Transport and Deposition of Flexible Fibers in Turbulent Flows," 19th Annual Conference of the American Association for Aerosol Research, AAAR 2000, St. Louis, MO, November 6-10, 2000.

H. Zhang, G. Ahmadi, R. Han and B.J. Greenspan, "Impact Breakup of Particle Pairs," 19th Annual Conference of the American Association for Aerosol Research, AAAR 2000, St. Louis, MO, November 6-10, 2000.

H. Zhang, G. Ahmadi, R. Han and B.J. Greenspan, "Breakup of Pairs of Attached Particles in Simple Shear Flows," 19th Annual Conference of the American Association for Aerosol Research, AAAR 2000, St. Louis, MO, November 6-10, 2000.

D.J. Schmidt, G. Ahmadi, and G. Schmidt, 'Lagrangian PDF: Application to Multiphase Spray Simulation," 4th International Conference on Multiphase Flows, ICMF' 2001, New Orleans, LA, May 27-June 1, 2001. 
C. Liu and G. Ahmadi, "Transport and Deposition of Atmospheric Particles Near a Building Model," 4th International Conference on Multiphase Flows, ICMF' 2001, New Orleans, LA, May 27-June 1, 2001.

M. Shams, G. Ahmadi and H. Rahimzadeh, "Flexible Fiber Motion in Turbulent Flows," 4th International Conference on Multiphase Flows, ICMF' 2001, New Orleans, LA, May 27-June $1,2001$.

\section{Students and Collaborators}

\$ X. Zhang, visiting scholar, Mech. \& Aero. Engineering, Clarkson University

\$ A.R. Mazaheri, graduate (Ph.D) student, Mech. \& Aero. Engineering, Clarkson University

\$ J. Cao, graduate (Ph.D) student, Mech. \& Aero. Engineering, Clarkson University

$\$ \quad$ H. Zhang, graduate (Ph.D) student, Mech. \& Aero. Engineering, Clarkson University

$\$ \quad$ C. He, graduate (Ph.D) student, Mech. \& Aero. Engineering, Clarkson University (Curently with Corning)

\$ W. Kvasnak, graduate (Ph.D) student, Mech. \& Aero. Engineering, Clarkson University (Currently with Pratt \& Whitney) 